\title{
Symmetric Closed Characteristics on Symmetric Compact Convex Hypersurfaces in $\mathbf{R}^{8}$
}

\author{
Hui Liu • Yiming Long • Wei Wang • \\ Ping'an Zhang
}

Received: 7 December 2014 / Accepted: 11 February 2015 / Published online: 13 March 2015

(C) School of Mathematical Sciences, University of Science and Technology of China and Springer-Verlag Berlin Heidelberg 2015

\begin{abstract}
Let $\Sigma$ be a $C^{3}$ compact symmetric convex hypersurface in $\mathbf{R}^{8}$. We prove that when $\Sigma$ carries exactly four geometrically distinct closed characteristics, then all of them must be symmetric. Due to the example of weakly non-resonant ellipsoids, our result is sharp.
\end{abstract}

Keywords Compact convex hypersurfaces - Symmetric closed characteristics · Hamiltonian systems $\cdot$ Morse theory $\cdot$ Index iteration theory

Mathematics Subject Classification $58 \mathrm{E} 05 \cdot 37 \mathrm{~J} 45 \cdot 37 \mathrm{C} 75$

H. Liu

Key Laboratory of Wu Wen-Tsun Mathematics, Chinese Academy of Sciences,

School of Mathematical Sciences, University of Science and Technology of China, Hefei 230026, Anhui, People's Republic of China

e-mail: huiliu@ustc.edu.cn

Y. Long $(\varangle)$

Chern Institute of Mathematics and LPMC, Nankai University, Tianjin 300071,

People's Republic of China

e-mail: longym@nankai.edu.cn

W. Wang

Key Laboratory of Pure and Applied Mathematics, School of Mathematical Science,

Peking University, Beijing 100871, People's Republic of China

e-mail: alexanderweiwang@gmail.com;wangwei@math.pku.edu.cn

P. Zhang

School of Mathematics and Statistics, Xidian University, Xi'an 710071,

Shaanxi, People's Republic of China

e-mail: pinganzhang@xidian.edu.cn 


\section{Introduction and Main Results}

In this paper, let $\Sigma$ be a fixed $C^{3}$ compact convex hypersurface in $\mathbf{R}^{2 n}$, i.e., $\Sigma$ is the boundary of a compact and strictly convex region $U_{\Sigma}$ in $\mathbf{R}^{2 n}$. We denote the set of all such hypersurfaces by $\mathcal{H}(2 n)$. Without loss of generality, we suppose $U$ contains the origin. We denote the set of all compact convex hypersurfaces which are symmetric with respect to the origin by $\mathcal{S H}(2 n)$, i.e., $\Sigma=-\Sigma$ for $\Sigma \in \mathcal{S H}(2 n)$. We consider closed characteristics $(\tau, y)$ on $\Sigma$, which are solutions of the following problem

$$
\left\{\begin{array}{l}
\dot{y}=J N_{\Sigma}(y) \\
y(\tau)=y(0)
\end{array}\right.
$$

where $J=\left(\begin{array}{cc}0 & -I_{n} \\ I_{n} & 0\end{array}\right), I_{n}$ is the identity matrix in $\mathbf{R}^{n}, \tau>0$ and $N_{\Sigma}(y)$ is the outward normal vector of $\Sigma$ at $y$ normalized by the condition $N_{\Sigma}(y) \cdot y=1$. Here $a \cdot b$ denotes the standard inner product of $a, b \in \mathbf{R}^{2 n}$. A closed characteristic $(\tau, y)$ is prime if $\tau$ is the minimal period of $y$. Two closed characteristics $(\tau, y)$ and $(\sigma, z)$ are geometrically distinct if $y(\mathbf{R}) \neq z(\mathbf{R})$. We denote by $\mathcal{J}(\Sigma)$ and $\widetilde{\mathcal{J}}(\Sigma)$ the set of all closed characteristics $(\tau, y)$ on $\Sigma$ with $\tau$ being the minimal period of $y$ and the set of all geometrically distinct ones, respectively. Note that $\mathcal{J}(\Sigma)=\{\theta \cdot y \mid \theta \in$ $S^{1}$, y is prime $\}$, while $\widetilde{\mathcal{J}}(\Sigma)=\mathcal{J}(\Sigma) / S^{1}$, where the natural $S^{1}$-action is defined by $\theta \cdot y(t)=y(t+\tau \theta), \quad \forall \theta \in S^{1}, t \in \mathbf{R}$. A closed characteristic $(\tau, y)$ on $\Sigma \in \mathcal{S H}(2 n)$ is symmetric if $y(\mathbf{R})=-y(\mathbf{R})$.

For the existence and multiplicity of geometrically distinct closed characteristics on compact convex hypersurfaces in $\mathbf{R}^{2 n}$, we refer to $[3,4,6,7,9-12,16,18,19]$ and references therein. Especially, In [7] of 2002, Liu, Long and Zhu proved that

$$
{ }^{\#} \tilde{\mathcal{J}}(\Sigma) \geq n, \quad \forall \Sigma \in \mathcal{S H}(2 n) .
$$

It solved a longstanding conjecture for closed characteristics on compact convex hypersurfaces in $\mathbf{R}^{2 n}$ when they are symmetric. In [14] of 2009, Wang proved that if there exist exactly $n$ closed characteristics on some $\Sigma \in \mathcal{S H}(2 n)$, then all of them are symmetric when $n=2,3$. Recently, Wang [16] proved that ${ }^{\#} \tilde{\mathcal{J}}(\Sigma) \geq 4$ for every $\Sigma \in \mathcal{H}(8)$. But in both [7,16] it is left open on whether the closed characteristics found are symmetric. Note that we have the following example of weakly non-resonant ellipsoid: Let $r=\left(r_{1}, \ldots, r_{n}\right)$ with $r_{i}>0$ for $1 \leq i \leq n$. Define

$$
\mathcal{E}_{n}(r)=\left\{z=\left(x_{1}, \ldots, x_{n}, y_{1}, \ldots, y_{n}\right) \in \mathbf{R}^{2 n} \mid \frac{1}{2} \sum_{i=1}^{n} \frac{x_{i}^{2}+y_{i}^{2}}{r_{i}^{2}}=1\right\},
$$

where $\frac{r_{i}}{r_{j}} \in \mathbf{R} \backslash \mathbf{Q}$ whenever $i \neq j$. In this case, the corresponding Hamiltonian system is linear and all the solutions of (1.1) can be computed explicitly. Thus, it is easy to verify that ${ }^{\#} \tilde{\mathcal{J}}\left(\mathcal{E}_{n}(r)\right)=n$ and all the closed characteristics on $\mathcal{E}_{n}(r)$ are symmetric. Note that it was conjectured by H. Hofer among others that ${ }^{\#} \tilde{\mathcal{J}}(\Sigma)=n$ or $+\infty$ holds 
for every $\Sigma \in \mathcal{H}(2 n)$. Here we tend to conjecture that for every $\Sigma \in \mathcal{S H}(2 n)$, all the closed characteristics on $\Sigma$ are symmetric when ${ }^{\#} \tilde{\mathcal{J}}(\Sigma)=n$.

Motivated by these results and conjectures, we confirm the symmetry conjecture for the case $n=4$ when ${ }^{*} \tilde{\mathcal{J}}(\Sigma)=4$ :

Theorem 1.1 For every $\Sigma \in \mathcal{S H}(8)$ satisfying ${ }^{\#} \tilde{\mathcal{J}}(\Sigma)=4$, all the closed characteristics on $\Sigma$ are symmetric.

In Sect. 2, we review briefly the equivariant Morse theory for closed characteristics on compact convex hypersurfaces in $\mathbf{R}^{2 n}$ developed in [18] and the Fadell-Rabinowitz index theory applied to the study of closed characteristics. In Sect. 3, we prove the main result using equivariant Morse theory, index iteration theory developed by Long and his coworkers, especially the common index jump theorem of Long and Zhu, and a commutative property for closed characteristics in the common index jump intervals discovered by Wang in [16] which played a crucial role in his proof of the existence of four geometrically distinct closed characteristics on every compact convex hypersurface in $\mathbf{R}^{8}$. Note that this commutative property was also used in [17].

In this paper, let $\mathbf{N}, \mathbf{N}_{0}, \mathbf{Z}, \mathbf{Q}, \mathbf{R}$, and $\mathbf{C}$ denote the sets of natural integers, nonnegative integers, integers, rational numbers, real numbers, and complex numbers respectively. Denote by $a \cdot b$ and $|a|$, the standard inner product and norm in $\mathbf{R}^{2 n}$. Denote by $\langle\cdot, \cdot\rangle$, and $\|\cdot\|$, the standard $L^{2}$-inner product and $L^{2}$-norm. For an $S^{1}$-space $X$, we denote by $X_{S^{1}}$ the homotopy quotient of $X$ module the $S^{1}$-action, i.e., $X_{S^{1}}=$ $S^{\infty} \times_{S^{1}} X$. In this paper, we use only Q-coefficients for all homological modules. For a $\mathbf{Z}_{m}$-space pair $(A, B)$, let $H_{*}(A, B)^{ \pm \mathbf{Z}_{m}}=\left\{\sigma \in H_{*}(A, B) \mid L_{*} \sigma= \pm \sigma\right\}$, where $L$ is a generator of the $\mathbf{Z}_{m}$-action. We denote by ${ }^{\#} A$ the number of elements in the set $A$ when it is finite.

\section{Equivariant Morse Theory for Closed Characteristics}

In this section, we fix a $\Sigma \in \mathcal{H}(2 n)$ and assume the following condition on $\Sigma$ :

(F) There exist only finitely many geometrically distinct closed characteristics $\left\{\left(\tau_{j}, y_{j}\right)\right\}_{1 \leq j \leq q}$ on $\Sigma$.

In this section, we review briefly the equivariant Morse theory for closed characteristics on $\Sigma$ developed in [18] and [13] which will be used in Sect. 4 of this paper. All the details of proofs can be found in [18] or [13].

Let $\hat{\tau}=\inf \left\{\tau_{j} \mid 1 \leq j \leq q\right\}$. Then by $\S 2$ of [18], for any $a>\hat{\tau}$, we can construct a function $\varphi_{a} \in C^{\infty}\left(\mathbf{R}, \mathbf{R}^{+}\right)$which has 0 as its unique critical point in $[0,+\infty)$ such that $\varphi_{a}$ is strictly convex for $t \geq 0$. Moreover, $\frac{\varphi_{a}^{\prime}(t)}{t}$ is strictly decreasing for $t>0$ together with $\lim _{t \rightarrow 0^{+}} \frac{\varphi_{a}^{\prime}(t)}{t}=1$ and $\varphi_{a}(0)=0=\varphi_{a}^{\prime}(0)$ (cf. Propositions 2.2-2.4 in [18]).

Let $j: \mathbf{R}^{2 n} \rightarrow \mathbf{R}$ be the gauge function of $\Sigma$, i.e., $j(\lambda x)=\lambda$ for $x \in \Sigma$ and $\lambda \geq 0$, then $j \in C^{3}\left(\mathbf{R}^{2 n} \backslash\{0\}, \mathbf{R}\right) \cap C^{0}\left(\mathbf{R}^{2 n}, \mathbf{R}\right)$ and $\Sigma=j^{-1}(1)$. Define the Hamiltonian function $H_{a}(x)=a \varphi_{a}(j(x))$ and consider the fixed period problem

$$
\left\{\begin{array}{l}
\dot{x}(t)=J H_{a}^{\prime}(x(t)), \\
x(1)=x(0) .
\end{array}\right.
$$


Then $H_{a} \in C^{3}\left(\mathbf{R}^{2 n} \backslash\{0\}, \mathbf{R}\right) \cap C^{1}\left(\mathbf{R}^{2 n}, \mathbf{R}\right)$ is strictly convex. Solutions of (2.1) are $x \equiv 0$ and $x=\rho y(\tau t)$ with $\frac{\varphi_{a}^{\prime}(\rho)}{\rho}=\frac{\tau}{a}$, where $(\tau, y)$ is a solution of (1.1). In particular, nonzero solutions of (2.1) are in one-to-one correspondence with solutions of (1.1) with period $\tau<a$.

Now, we use the Clarke-Ekeland dual action principle to transform (2.1) to a variational problem and use variational methods to study the problem. As usual, let $G_{a}$ be the Fenchel transform of $H_{a}$ defined by $G_{a}(y)=\sup \left\{x \cdot y-H_{a}(x) \mid x \in \mathbf{R}^{2 n}\right\}$. Then $G_{a} \in C^{2}\left(\mathbf{R}^{2 n} \backslash\{0\}, \mathbf{R}\right) \cap C^{1}\left(\mathbf{R}^{2 n}, \mathbf{R}\right)$ is strictly convex. Let

$$
L_{0}^{2}\left(S^{1}, \mathbf{R}^{2 n}\right)=\left\{u \in L^{2}\left([0,1], \mathbf{R}^{2 n}\right) \mid \int_{0}^{1} u(t) \mathrm{d} t=0\right\} .
$$

Define a linear operator $M: L_{0}^{2}\left(S^{1}, \mathbf{R}^{2 n}\right) \rightarrow L_{0}^{2}\left(S^{1}, \mathbf{R}^{2 n}\right)$ by $\frac{\mathrm{d}}{\mathrm{d} t} M u(t)=u(t)$, $\int_{0}^{1} M u(t) \mathrm{d} t=0$. The dual action functional on $L_{0}^{2}\left(S^{1}, \mathbf{R}^{2 n}\right)$ is defined by

$$
\Psi_{a}(u)=\int_{0}^{1}\left(\frac{1}{2} J u \cdot M u+G_{a}(-J u)\right) \mathrm{d} t .
$$

Then the functional $\Psi_{a} \in C^{1,1}\left(L_{0}^{2}\left(S^{1}, \mathbf{R}^{2 n}\right), \mathbf{R}\right)$ is bounded from below and satisfies the Palais-Smale condition. Suppose $x$ is a solution of (2.1). Then $u=\dot{x}$ is a critical point of $\Psi_{a}$. Conversely, suppose $u$ is a critical point of $\Psi_{a}$. Then there exists a unique $\xi \in \mathbf{R}^{2 n}$ such that $M u-\xi$ is a solution of (2.1). In particular, solutions of (2.1) are in one-to-one correspondence with critical points of $\Psi_{a}$. Moreover, $\Psi_{a}(u)<0$ for every critical point $u \neq 0$ of $\Psi_{a}$.

Suppose $u$ is a nonzero critical point of $\Psi_{a}$. Then following [2], the formal Hessian of $\Psi_{a}$ at $u$ is defined by

$$
Q_{a}(v, v)=\int_{0}^{1}\left(J v \cdot M v+G_{a}^{\prime \prime}(-J u) J v \cdot J v\right) \mathrm{d} t,
$$

which defines an orthogonal splitting $L_{0}^{2}\left(S^{1}, \mathbf{R}^{2 n}\right)=E_{-} \oplus E_{0} \oplus E_{+}$of $L_{0}^{2}\left(S^{1}, \mathbf{R}^{2 n}\right)$ into negative, zero, and positive subspaces. The index of $u$ is defined by $i(u)=\operatorname{dim} E_{-}$ and the nullity of $u$ is defined by $v(u)=\operatorname{dim} E_{0}$. Let $u=\dot{x}$ be the critical point of $\Psi_{a}$ such that $x$ corresponds to a closed characteristic $(\tau, y)$ on $\Sigma$. Then the index $i(u)$ and the nullity $v(u)$ defined above coincide with the Ekeland indices defined by Ekeland in $[1,2]$. In particular, $1 \leq v(u) \leq 2 n-1$ always holds.

We have a natural $S^{1}$-action on $L_{0}^{2}\left(S^{1}, \mathbf{R}^{2 n}\right)$ defined by $\theta \cdot u(t)=u(\theta+t)$ for all $\theta \in S^{1}$ and $t \in \mathbf{R}$. Clearly $\Psi_{a}$ is $S^{1}$-invariant. For any $\kappa \in \mathbf{R}$, we denote by

$$
\Lambda_{a}^{\kappa}=\left\{w \in L_{0}^{2}\left(S^{1}, \mathbf{R}^{2 n}\right) \mid \Psi_{a}(w) \leq \kappa\right\}
$$

For a critical point $u$ of $\Psi_{a}$, we denote by

$$
\Lambda_{a}(u)=\Lambda_{a}^{\Psi_{a}(u)}=\left\{w \in L_{0}^{2}\left(S^{1}, \mathbf{R}^{2 n}\right) \mid \Psi_{a}(w) \leq \Psi_{a}(u)\right\} .
$$


Clearly, both sets are $S^{1}$-invariant. Since the $S^{1}$-action preserves $\Psi_{a}$, if $u$ is a critical point of $\Psi_{a}$, then the whole orbit $S^{1} \cdot u$ is formed by critical points of $\Psi_{a}$. Denote by $\operatorname{crit}\left(\Psi_{a}\right)$ the set of critical points of $\Psi_{a}$. Note that by the condition $(\mathrm{F})$, the number of critical orbits of $\Psi_{a}$ is finite. Hence as usual we can make the following definition.

Definition 2.1 Suppose $u$ is a nonzero critical point of $\Psi_{a}$ and $\mathcal{N}$ is an $S^{1}$-invariant open neighborhood of $S^{1} \cdot u$ such that $\operatorname{crit}\left(\Psi_{a}\right) \cap\left(\Lambda_{a}(u) \cap \mathcal{N}\right)=S^{1} \cdot u$. Then the $S^{1}$-critical modules of $S^{1} \cdot u$ are defined by

$$
C_{S^{1}, k}\left(\Psi_{a}, S^{1} \cdot u\right)=H_{k}\left(\left(\Lambda_{a}(u) \cap \mathcal{N}\right)_{S^{1}}, \quad\left(\left(\Lambda_{a}(u) \backslash S^{1} \cdot u\right) \cap \mathcal{N}\right)_{S^{1}}\right), \quad k \in \mathbf{Z} .
$$

We have the following proposition for critical modules.

Proposition 2.2 (Proposition 3.2 of [18]) The critical module $C_{S^{1}, k}\left(\Psi_{a}, S^{1} \cdot u\right.$ ) is independent of $a$ in the sense that if $x_{i}$ are solutions of (2.1) with Hamiltonian functions $H_{a_{i}}(x) \equiv a_{i} \varphi_{a_{i}}(j(x))$ for $i=1$ and 2 , respectively such that both $x_{1}$ and $x_{2}$ correspond to the same closed characteristic $(\tau, y)$ on $\Sigma$. Then we have

$$
C_{S^{1}, k}\left(\Psi_{a_{1}}, S^{1} \cdot \dot{x}_{1}\right) \cong C_{S^{1}, k}\left(\Psi_{a_{2}}, S^{1} \cdot \dot{x}_{2}\right), \quad \forall k \in \mathbf{Z}
$$

Now let $u \neq 0$ be a critical point of $\Psi_{a}$ with multiplicity $\operatorname{mul}(u)=m$, i.e., $u$ corresponds to a closed characteristic $(m \tau, y) \subset \Sigma$ with $(\tau, y)$ being prime. Hence $u\left(t+\frac{1}{m}\right)=u(t)$ holds for all $t \in \mathbf{R}$. For any $p \in \mathbf{N}$, denote by $u^{p}$ the unique critical point of $\Psi_{a}$ corresponding to $(p m \tau, y)$. By Sect. 3 of [18], we can construct a $\mathbf{Z}_{p m^{-}}$ invariant local characteristic manifold $W\left(u^{p}\right)$ at $u^{p}$ with dimension $v\left(u^{p}\right)-1$ using a finite-dimensional reduction and Gromoll-Meyer theory. Then we have the following proposition.

Proposition 2.3 (Proposition 3.10 of [18]) Let $u \neq 0$ be a critical point of $\Psi_{a}$ with $\operatorname{mul}(u)=1$. Then for all $p \in \mathbf{N}$ and $j \in \mathbf{Z}$, we have

$$
\begin{aligned}
C_{S^{1}, j}\left(\Psi_{a}, S^{1} \cdot u^{p}\right) \cong & \left(H _ { j - i ( u ^ { p } ) } \left(W\left(u^{p}\right)\right.\right. \\
& \left.\left.\cap \Lambda_{a}\left(u^{p}\right),\left(W\left(u^{p}\right) \backslash\left\{u^{p}\right\}\right) \cap \Lambda_{a}\left(u^{p}\right)\right)\right)^{\beta\left(u^{p}\right) \mathbf{Z}_{p}},
\end{aligned}
$$

where $\beta\left(u^{p}\right)=(-1)^{i\left(u^{p}\right)-i(u)}$. Thus

$$
C_{S^{1}, j}\left(\Psi_{a}, S^{1} \cdot u^{p}\right)=0, \text { for } j<i\left(u^{p}\right) \text { or } j>i\left(u^{p}\right)+v\left(u^{p}\right)-1 .
$$

In particular, if $u^{p}$ is non-degenerate, i.e., $v\left(u^{p}\right)=1$, then

$$
C_{S^{1}, j}\left(\Psi_{a}, S^{1} \cdot u^{p}\right)=\left\{\begin{array}{ll}
\mathbf{Q}, & \text { if } j=i\left(u^{p}\right) \\
0, & \text { otherwise }
\end{array} \text { and } \beta\left(u^{p}\right)=1,\right.
$$

We make the following definition. 
Definition 2.4 Let $u \neq 0$ be a critical point of $\Psi_{a}$ with $\operatorname{mul}(u)=1$. Then for all $p \in \mathbf{N}$ and $l \in \mathbf{Z}$, let

$$
\begin{aligned}
k_{l, \pm 1}\left(u^{p}\right) & =\operatorname{dim}\left(H_{l}\left(W\left(u^{p}\right) \cap \Lambda_{a}\left(u^{p}\right),\left(W\left(u^{p}\right) \backslash\left\{u^{p}\right\}\right) \cap \Lambda_{a}\left(u^{p}\right)\right)\right)^{ \pm \mathbf{Z}_{p}} \\
k_{l}\left(u^{p}\right) & =\operatorname{dim}\left(H_{l}\left(W\left(u^{p}\right) \cap \Lambda_{a}\left(u^{p}\right),\left(W\left(u^{p}\right) \backslash\left\{u^{p}\right\}\right) \cap \Lambda_{a}\left(u^{p}\right)\right)\right)^{\beta\left(u^{p}\right) \mathbf{Z}_{p}} .
\end{aligned}
$$

$k_{l}\left(u^{p}\right)$ 's are called critical type numbers of $u^{p}$.

We have the following periodic property for critical type numbers.

Proposition 2.5 (Lemma 3.12 of [18]) Let $u \neq 0$ be a critical point of $\Psi_{a}$ with $\operatorname{mul}(u)=1$. Suppose that $v\left(u^{m}\right)=v\left(u^{p m}\right)$ for some $p, m \in \mathbf{N}$, then we have $k_{l, \pm 1}\left(u^{m}\right)=k_{l, \pm 1}\left(u^{p m}\right)$ for all $l \in \mathbf{Z}$.

Proposition 2.6 (Proposition 3.13 of [18]) Let $u \neq 0$ be a critical point of $\Psi_{a}$ with $\operatorname{mul}(u)=1$. Then there exists a minimal $K(u) \in \mathbf{N}$ such that

$$
v\left(u^{p+K(u)}\right)=v\left(u^{p}\right), \quad i\left(u^{p+K(u)}\right)-i\left(u^{p}\right) \in 2 \mathbf{Z} .
$$

Moreover, we have $k_{l}\left(u^{p+K(u)}\right)=k_{l}\left(u^{p}\right)$ for all $p \in \mathbf{N}$ and $l \in \mathbf{Z}$.

In fact, denote by $\gamma_{y}$ the associated symplectic path of $(\tau, y)$, where $(\tau, y)$ is the closed characteristic corresponding to $u$. Suppose $\lambda_{i}=e^{ \pm \frac{r_{i}}{s_{i}} 2 \pi \sqrt{-1}}$ the eigenvalues of $\gamma_{y}(\tau)$ possessing rotation angles which are rational multiple of $2 \pi$ with $r_{i}, s_{i} \in \mathbf{N}$ and $\left(r_{i}, s_{i}\right)=1$ for $1 \leq i \leq k$. Let $K^{\prime}(u)$ be the least common multiple of $s_{1}, \ldots, s_{k}$. Then we have $v\left(u^{p+K^{\prime}(u)}\right)=v\left(u^{p}\right)$ for all $p \in \mathbf{N}$. By Theorem 3.1 below and Theorem 9.3.4 of [9], we have $i\left(u^{m+2}\right)-i\left(u^{m}\right) \in 2 \mathbf{Z}$ for any $m \in \mathbf{N}$. Hence we have

$$
K(u)= \begin{cases}2 K^{\prime}(u) & \text { if } i\left(u^{2}\right)-i(u) \in 2 \mathbf{Z}+1 \text { and } K^{\prime}(u) \in 2 \mathbf{N}-1, \\ K^{\prime}(u) & \text { otherwise }\end{cases}
$$

For a prime closed characteristic $(\tau, y)$ on $\Sigma$, we denote by $y^{m} \equiv(m \tau, y)$ the $m$-th iteration of $y$ for $m \in \mathbf{N}$. Let $a>\tau$ be large enough and choose $\varphi_{a}$ as above. Determine $\rho$ uniquely by $\frac{\varphi_{a}^{\prime}(\rho)}{\rho}=\frac{\tau}{a}$. Let $x=\rho y(\tau t)$ and $u=\dot{x}$. Then we define the index $i\left(y^{m}\right)$ and nullity $v\left(y^{m}\right)$ of $(m \tau, y)$ for $m \in \mathbf{N}$ by

$$
i\left(y^{m}\right)=i\left(u^{m}\right), \quad v\left(y^{m}\right)=v\left(u^{m}\right) .
$$

These indices are independent of $a$ when $a$ tends to infinity. Now the mean index of $(\tau, y)$ is defined by

$$
\hat{i}(y)=\lim _{m \rightarrow \infty} \frac{i\left(y^{m}\right)}{m} .
$$

Note that $\hat{i}(y)>2$ always holds which was proved by Ekeland and Hofer in [3] of 1987 (cf. Corollary 8.3.2 and Lemma 15.3.2 of [9] for a different proof). 
By Proposition 2.2, we can define the critical type numbers $k_{l}\left(y^{m}\right)$ of $y^{m}$ to be $k_{l}\left(u^{m}\right)$, where $u^{m}$ is the critical point of $\Psi_{a}$ corresponding to $y^{m}$. We also define $K(y)=K(u)$. Then we have the following

Proposition 2.7 (Proposition 2.6 of [13]) We have $k_{l}\left(y^{m}\right)=0$ for $l \notin\left[0, v\left(y^{m}\right)-1\right]$ and it can take only values 0 or 1 when $l=0$ or $l=v\left(y^{m}\right)-1$. Moreover, the following properties hold:

(i) $k_{0}\left(y^{m}\right)=1$ implies $k_{l}\left(y^{m}\right)=0$ for $1 \leq l \leq v\left(y^{m}\right)-1$.

(ii) $k_{v\left(y^{m}\right)-1}\left(y^{m}\right)=1$ implies $k_{l}\left(y^{m}\right)=0$ for $0 \leq l \leq v\left(y^{m}\right)-2$.

(iii) $k_{l}\left(y^{m}\right) \geq 1$ for some $1 \leq l \leq v\left(y^{m}\right)-2$ implies $k_{0}\left(y^{m}\right)=k_{v\left(y^{m}\right)-1}\left(y^{m}\right)=0$.

(iv) If $i\left(y^{m}\right)-i(y) \in 2 \mathbf{Z}+1$ for some $m \in \mathbf{N}$, then $k_{0}\left(y^{m}\right)=0$.

Let $\Psi_{a}$ be the functional defined by (2.3) for some $a \in \mathbf{R}$ large enough and let $\varepsilon>0$ be small enough such that $[-\varepsilon,+\infty) \backslash\{0\}$ contains no critical values of $\Psi_{a}$. Denote by $I_{a}$ the greatest integer in $\mathbf{N}_{0}$ such that $I_{a}<i(\tau, y)$ hold for all closed characteristics $(\tau, y)$ on $\Sigma$ with $\tau \geq a$. Then by pp. 447 and 448 of [18], we have

$$
H_{S^{1}, i}\left(\Lambda_{a}^{\infty}\right) \cong H_{S^{1}, i}\left(\Lambda_{a}^{\infty}\right) \cong H_{i}\left(C P^{\infty}\right), \quad \forall i<I_{a}
$$

For any $i \in \mathbf{Z}$, let

$$
M_{i}\left(\Lambda_{a}^{-\varepsilon}\right)=\sum_{1 \leq j \leq q, 1 \leq m_{j}<a / \tau_{j}} \operatorname{dim} C_{S^{1}, i}\left(\Psi_{a}, S^{1} \cdot u_{j}^{m_{j}}\right)
$$

Then the equivariant Morse inequalities for the space $\Lambda_{a}^{-\varepsilon}$ yield

$$
\begin{gathered}
M_{i}\left(\Lambda_{a}^{-\varepsilon}\right) \geq b_{i}\left(\Lambda_{a}^{-\varepsilon}\right), \\
M_{i}\left(\Lambda_{a}^{-\varepsilon}\right)-M_{i-1}\left(\Lambda_{a}^{-\varepsilon}\right)+\cdots+(-1)^{i} M_{0}\left(\Lambda_{a}^{-\varepsilon}\right) \\
\geq b_{i}\left(\Lambda_{a}^{-\varepsilon}\right)-b_{i-1}\left(\Lambda_{a}^{-\varepsilon}\right)+\cdots+(-1)^{i} b_{0}\left(\Lambda_{a}^{-\varepsilon}\right),
\end{gathered}
$$

for $i \in \mathbf{Z}$, where $b_{i}\left(\Lambda_{a}^{-\varepsilon}\right)=\operatorname{dim} H_{S^{1}, i}\left(\Lambda_{a}^{-\varepsilon}\right)$. Now we have the following Morse inequalities for closed characteristics.

Theorem 2.8 (Theorem 2.8 of [13]) Suppose $\Sigma \in \mathcal{H}(2 n)$ satisfy ${ }^{\#} \mathcal{J}(\Sigma)<+\infty$. Denote all the geometrically distinct closed characteristics on $\Sigma$ by $\left\{\left(\tau_{j}, y_{j}\right)\right\}_{1 \leq j \leq k}$. Let

$$
\begin{aligned}
M_{i}= & \lim _{a \rightarrow+\infty} M_{i}\left(\Lambda_{a}^{-\varepsilon}\right), \quad \forall i \in \mathbf{Z}, \\
& \\
b_{i}= & \lim _{\substack{a \rightarrow+\infty \\
\varepsilon \rightarrow 0}} b_{i}\left(\Lambda_{a}^{-\varepsilon}\right)= \begin{cases}1, & \text { if } i \in 2 \mathbf{N}_{0}, \\
0, & \text { otherwise. }\end{cases}
\end{aligned}
$$


Then we have

$$
\begin{aligned}
M_{i} & \geq b_{i}, \quad \forall i \in \mathbf{Z}, \\
M_{i}-M_{i-1}+\cdots+(-1)^{i} M_{0} & \geq b_{i}-b_{i-1}+\cdots+(-1)^{i} b_{0}, \quad \forall i \in \mathbf{Z} .
\end{aligned}
$$

Recall that for a principal $U(1)$-bundle $E \rightarrow B$, the Fadell-Rabinowitz index (cf. [5]) of $E$ is defined to be $\sup \left\{k \mid c_{1}(E)^{k-1} \neq 0\right\}$, where $c_{1}(E) \in H^{2}(B, \mathbf{Q})$ is the first rational Chern class. For a $U(1)$-space, i.e., a topological space $X$ with a $U(1)$-action, the Fadell-Rabinowitz index is defined to be the index of the bundle $X \times S^{\infty} \rightarrow X \times_{U(1)} S^{\infty}$, where $S^{\infty} \rightarrow C P^{\infty}$ is the universal $U$ (1)-bundle.

As in P. 199 of [2], choose some $\alpha \in(1,2)$ and associate with $U_{\Sigma}$ a convex function $H$ such that $H(\lambda x)=\lambda^{\alpha} H(x)$ for $\lambda \geq 0$. Consider the fixed period problem

$$
\left\{\begin{array}{l}
\dot{x}(t)=J H^{\prime}(x(t)) \\
x(1)=x(0)
\end{array}\right.
$$

Define

$$
L_{0}^{\frac{\alpha}{\alpha-1}}\left(S^{1}, \mathbf{R}^{2 n}\right)=\left\{u \in L^{\frac{\alpha}{\alpha-1}}\left(S^{1}, \mathbf{R}^{2 n}\right) \mid \int_{0}^{1} u \mathrm{~d} t=0\right\} .
$$

The corresponding Clarke-Ekeland dual action functional is defined by

$$
\Phi(u)=\int_{0}^{1}\left(\frac{1}{2} J u \cdot M u+H^{*}(-J u)\right) \mathrm{d} t, \quad \forall u \in L_{0}^{\frac{\alpha}{\alpha-1}}\left(S^{1}, \mathbf{R}^{2 n}\right),
$$

where $M u$ is defined by $\frac{\mathrm{d}}{\mathrm{d} t} M u(t)=u(t)$ and $\int_{0}^{1} M u(t) \mathrm{d} t=0, H^{*}$ is the Fenchel transform of $H$ defined above.

For any $\kappa \in \mathbf{R}$, we denote by

$$
\Phi^{\kappa-}=\left\{u \in L_{0}^{\frac{\alpha}{\alpha-1}}\left(S^{1}, \mathbf{R}^{2 n}\right) \mid \Phi(u)<\kappa\right\} .
$$

Then as in P. 218 of [2], we define

$$
c_{i}=\inf \left\{\delta \in \mathbf{R} \mid \hat{I}\left(\Phi^{\delta-}\right) \geq i\right\}
$$

where $\hat{I}$ is the Fadell-Rabinowitz index given above. Then by Proposition 3 in P. 218 of [2], we have

Proposition 2.9 Every $c_{i}$ is a critical value of $\Phi$. If $c_{i}=c_{j}$ for some $i<j$, then there are infinitely many geometrically distinct closed characteristics on $\Sigma$.

As in Definition 2.1, we define the following 
Definition 2.10 Suppose $u$ is a nonzero critical point of $\Phi$, and $\mathcal{N}$ is an $S^{1}$-invariant open neighborhood of $S^{1} \cdot u$ such that $\operatorname{crit}(\Phi) \cap(\Lambda(u) \cap \mathcal{N})=S^{1} \cdot u$. Then the $S^{1}$-critical modules of $S^{1} \cdot u$ is defined by

$$
C_{S^{1}, k}\left(\Phi, S^{1} \cdot u\right)=H_{k}\left((\Lambda(u) \cap \mathcal{N})_{S^{1}},\left(\left(\Lambda(u) \backslash S^{1} \cdot u\right) \cap \mathcal{N}\right)_{S^{1}}\right), \quad k \in \mathbf{Z},
$$

where $\Lambda(u)=\left\{w \in L_{0}^{\frac{\alpha}{\alpha-1}}\left(S^{1}, \mathbf{R}^{2 n}\right) \mid \Phi(w) \leq \Phi(u)\right\}$.

Comparing with Theorem 4 in P. 219 of [2], we have the following

Proposition 2.11 (Proposition 3.5 of [13]) For every $i \in \mathbf{N}$, there exists a point $u \in L_{0}^{\frac{\alpha}{\alpha-1}}\left(S^{1}, \mathbf{R}^{2 n}\right)$ such that

$$
\Phi^{\prime}(u)=0, \quad \Phi(u)=c_{i}, \quad C_{S^{1}, 2(i-1)}\left(\Phi, S^{1} \cdot u\right) \neq 0
$$

The next proposition implies that $\Psi_{a}$ and $\Phi$ have isomorphic critical modules at corresponding critical points, thus we can compute the critical modules of $\Phi$ via that of $\Psi_{a}$.

Proposition 2.12 (Proposition 3.6 of [13]) Suppose $u$ is the critical point of $\Phi$ found in Proposition 2.11. Then we have

$$
C_{S^{1}, k}\left(\Psi_{a}, S^{1} \cdot u_{a}\right) \cong C_{S^{1}, k}\left(\Phi, S^{1} \cdot u\right), \quad \forall k \in \mathbf{Z},
$$

where $\Psi_{a}$ is given by (2.3) and $u_{a} \in L_{0}^{2}\left(S^{1}, \mathbf{R}^{2 n}\right)$ is its critical point corresponding to $u$ in the natural sense.

\section{Proof of the Main Theorem}

In this section, we give the proof of the main theorem.

The following theorem relates the Morse index defined in Sect. 2 to the Maslov-type index.

Theorem 3.1 (cf. Lemma 1.1 of [10], Theorem 15.1.1 of [9]) Suppose ( $\tau, y) \in \mathcal{J}(\Sigma)$. Then we have

$$
i\left(y^{m}\right) \equiv i(m \tau, y)=i(y, m)-n, \quad v\left(y^{m}\right) \equiv v(m \tau, y)=v(y, m), \quad \forall m \in \mathbf{N},
$$

where $i(y, m)$ and $v(y, m)$ are the Maslov-type index and nullity of $(m \tau, y)$ (cf. Sect. 5.4 of [9]).

As Definition 1.1 of [10], we define the following: 
Definition 3.2 For $\alpha \in(1,2)$, we define a map $\varrho_{n}: \mathcal{H}(2 n) \rightarrow \mathbf{N} \cup\{+\infty\}$

$$
\varrho_{n}(\Sigma)= \begin{cases}+\infty, & \text { if }{ }^{\#} \mathcal{V}(\Sigma, \alpha)=+\infty \\ \min \left\{\left[\frac{i(y, 1)+2 S^{+}(y)-v(y, 1)+n}{2}\right] \mid(\tau, y) \in \mathcal{V}_{\infty}(\Sigma, \alpha)\right\}, & \text { if }{ }^{\#} \mathcal{V}(\Sigma, \alpha)<+\infty\end{cases}
$$

where $\mathcal{V}(\Sigma, \alpha)$ and $\mathcal{V}_{\infty}(\Sigma, \alpha)$ are variationally visible and infinite variationally visible sets, respectively, given by Definition 1.4 of [10] (cf. Definition 15.3.3 of [9]).

In the rest of this paper, we fix a $\Sigma \in \mathcal{S H}(2 n)$. We have the following property, cf. Lemma 4.2 of [7].

Lemma 3.3 Suppose $(\tau, y) \in \mathcal{J}(\Sigma)$, then $(\tau,-y) \in \mathcal{J}(\Sigma)$ and either $\mathcal{O}(y)=$ $\mathcal{O}(-y)$ or $\mathcal{O}(y) \cap \mathcal{O}(-y)=\emptyset$, where $\mathcal{O}( \pm y)=\{ \pm y(t) \mid t \in \mathbf{R}\}$. Moreover, if $\mathcal{O}(y) \cap \mathcal{O}(-y) \neq \varnothing$, then we have

$$
y(t)=-y\left(t+\frac{\tau}{2}\right), \quad \forall t \in \mathbf{R} .
$$

In the following, we call a closed characteristic $(\tau, y)$ on $\Sigma \in \mathcal{S H}(2 n)$ symmetric if $\mathcal{O}(y) \cap \mathcal{O}(-y) \neq \emptyset$, asymmetric if $\mathcal{O}(y) \cap \mathcal{O}(-y)=\emptyset$. Thus if $(\tau, y)$ is asymmetric, then $(\tau, y)$ and $(\tau,-y)$ are geometrically distinct; if $(\tau, y)$ is symmetric, then $(\tau, y)$ and $(\tau,-y)$ are geometrically the same.

By Theorem 3.3 of [14], we have

Lemma 3.4 Suppose $\Sigma \in \mathcal{S H}(2 n)$ and $(\tau, y) \in \mathcal{J}(\Sigma)$. Then we have

$$
\begin{aligned}
\left(i\left(y^{m}\right), v\left(y^{m}\right)\right) & =\left(i\left((-y)^{m}\right), \quad v\left((-y)^{m}\right)\right), \\
\Phi\left(u^{m}\right) & =\Phi\left((-u)^{m}\right), \quad \forall m \in \mathbf{N}, \\
C_{S^{1}, k}\left(\Psi_{a}, S^{1} \cdot u^{m}\right) & \cong C_{S^{1}, k}\left(\Psi_{a}, S^{1} \cdot(-u)^{m}\right), \quad \forall m \in \mathbf{N}, \quad \forall k \in \mathbf{Z},
\end{aligned}
$$

where we denote simply by $( \pm u)^{m}$ the critical point of $\Phi$ or $\Psi_{a}$ corresponding to $( \pm y)^{m}$.

The following lemma for symmetric closed characteristics is directly from Theorem 1.1 of [15]. ${ }^{\#} \mathcal{J}_{S}(\Sigma)$ represents the number of symmetric closed characteristics on $\Sigma$.

Lemma 3.5 ${ }^{\#} \mathcal{J}_{s}(\Sigma) \geq 2$ for any $\Sigma \in \mathcal{S H}(2 n)$.

For symmetric closed characteristics, we also have:

Lemma 3.6 (cf. Lemma 4.1 of [7], Lemma 15.6.4 of [9]) Suppose ( $\tau, y) \in \mathcal{J}(\Sigma)$ is a symmetric closed characteristic on $\Sigma \in \mathcal{S H}(2 n)$. Then we have

$$
i(y, 1)+2 S^{+}(y)-v(y, 1) \geq n,
$$

where $S^{+}(y)$ is the splitting number defined in Sect. 9 of [9]. 
In the following, we fix $n=4$ and give the proof of Theorem 1.1.

Note that ${ }^{\#} \tilde{\mathcal{J}}(\Sigma)=4$, we denote by $\left\{\left(\tau_{j}, y_{j}\right)\right\}_{1 \leq j \leq 4}$ all the geometrically distinct closed characteristics on $\Sigma$, and by $\gamma_{j} \equiv \gamma_{y_{j}}$ the associated symplectic path of $\left(\tau_{j}, y_{j}\right)$ on $\Sigma$ for $1 \leq j \leq 4$. Then by Lemma 1.3 of [10] (cf. Lemma 15.2.4 of [9]), there exist $P_{j} \in S p(8)$ and $M_{j} \in S p(6)$ such that

$$
\gamma_{j}\left(\tau_{j}\right)=P_{j}^{-1}\left(N_{1}(1,1) \diamond M_{j}\right) P_{j}, \quad \forall 1 \leq j \leq 4,
$$

where $N_{1}(1, b)=\left(\begin{array}{ll}1 & b \\ 0 & 1\end{array}\right)$ for $b \in \mathbf{R}$.

By Corollary 1.2 of [10] (see also Corollary 15.1.4 of [9]), we have $i\left(y_{j}, 1\right) \geq 4$ for $1 \leq j \leq 4$. Note that $e\left(\gamma_{j}\left(\tau_{j}\right)\right) \leq 8$ for $1 \leq j \leq 4$, where $e(M)$ is the total algebraic multiplicity of all eigenvalues of $M$ on the unit circle $\mathbf{U}=\{z \in \mathbf{C}|| z \mid=1\}$ in the complex plane C. Hence Theorem 2.3 of [10] (see also Theorem 10.2.4 of [9]) yields

$$
\begin{aligned}
i\left(y_{j}, m\right)+v\left(y_{j}, m\right) & \leq i\left(y_{j}, m+1\right)-i\left(y_{j}, 1\right)+\frac{e\left(\gamma_{j}\left(\tau_{j}\right)\right)}{2}-1 \\
& \leq i\left(y_{j}, m+1\right)-1, \quad \forall m \in \mathbf{N}, \quad 1 \leq j \leq 4 .
\end{aligned}
$$

Since $\hat{i}\left(y_{j}\right)>2$ for $1 \leq j \leq 4$, we can use the common index jump theorem of Long and Zhu (Theorems 4.3 and 4.4 of [10], Theorems 11.2.1 and 11.2.2 of [9]), we can obtain that there are some $\left(T, m_{1}, m_{2}, m_{3}, m_{4}\right) \in \mathbf{N}^{5}$ such that the following hold:

$$
\begin{aligned}
i\left(y_{j}, 2 m_{j}\right) & \geq 2 T-\frac{e\left(\gamma_{j}\left(\tau_{j}\right)\right)}{2} \geq 2 T-4, \\
i\left(y_{j}, 2 m_{j}\right)+v\left(y_{j}, 2 m_{j}\right) & \leq 2 T+\frac{e\left(\gamma_{j}\left(\tau_{j}\right)\right)}{2}-1 \leq 2 T+3, \\
i\left(y_{j}, 2 m_{j}+1\right) & =2 T+i\left(y_{j}, 1\right), \\
i\left(y_{j}, 2 m_{j}-1\right)+v\left(y_{j}, 2 m_{j}-1\right) & =2 T-\left(i\left(y_{j}, 1\right)+2 S_{\gamma_{j}\left(\tau_{j}\right)}^{+}(1)-v\left(y_{j}, 1\right)\right) .
\end{aligned}
$$

More precisely, by (4.10), (4.40) and (4.41) in [10], we have

$$
m_{k}=\left(\left[\frac{T}{M \hat{i}\left(y_{k}, 1\right)}\right]+\chi_{k}\right) M, \quad 1 \leq k \leq 4,
$$

where $\chi_{k}=0$ or 1 for $1 \leq k \leq 4$ and $\frac{M \theta}{\pi} \in \mathbf{Z}$ whenever $e^{\sqrt{-1} \theta} \in \sigma\left(M_{k}\right)$ and $\frac{\theta}{\pi} \in \mathbf{Q}$ for some $1 \leq k \leq 4$. In fact, by (4.40) and (4.41) of [10], let $\mu_{i}=\sum_{\theta \in(0,2 \pi)} S_{M_{i}}^{-}\left(e^{\sqrt{-1} \theta}\right)$ for $1 \leq i \leq 4$ and $\alpha_{i, j}=\frac{\theta_{j}}{\pi}$, where $e^{\sqrt{-1} \theta_{j}} \in \sigma\left(M_{i}\right)$ for $1 \leq j \leq \mu_{i}$ and $1 \leq i \leq 4$. As in (4.21) of [10], let $h=4+\sum_{1 \leq i \leq 4} \mu_{i}$ and 


$$
\begin{aligned}
v= & \frac{1}{M \hat{i}\left(y_{1}, 1\right)}, \ldots, \frac{1}{M \hat{i}\left(y_{4}, 1\right)}, \frac{\alpha_{1,1}}{\hat{i}\left(y_{1}, 1\right)}, \frac{\alpha_{1,2}}{\hat{i}\left(y_{1}, 1\right)}, \ldots \frac{\alpha_{1, \mu_{1}}}{\hat{i}\left(y_{1}, 1\right)}, \frac{\alpha_{2,1}}{\hat{i}\left(y_{2}, 1\right)} \\
& \left., \ldots, \frac{\alpha_{4, \mu_{4}}}{\hat{i}\left(y_{4}, 1\right)}\right) .
\end{aligned}
$$

Then by (4.22) of [10], the common index jump theorem is equivalent to find a vertex

$$
\chi=\left(\chi_{1}, \ldots, \chi_{4}, \chi_{1,1}, \chi_{1,2}, \ldots, \chi_{1, \mu_{1}}, \chi_{2,1}, \ldots, \chi_{4, \mu_{4}}\right)
$$

of the cube $[0,1]^{h}$ and infinitely many integers $T \in \mathbf{N}$ such that

$$
|\{T v\}-\chi|<\epsilon
$$

for any given $\epsilon$ small enough.

From Lemma 3.5, we know that there exist at least two geometrically distinct symmetric closed characteristics, without loss of generality, we assume $\left(\tau_{j}, y_{j}\right)_{1 \leq j \leq 2}$ are symmetric. Now we prove Theorem 1.1 by contradiction. Thus in the following, by Lemma 3.3 we suppose $\left(\tau_{4}, y_{4}\right)=\left(\tau_{3},-y_{3}\right)$ which are asymmetric. Since otherwise we would have at least five geometrically distinct closed characteristics on $\Sigma$.

By Theorem 5.1 of [10](see also Theorem 15.4 .3 of [9]), we have $\mathcal{V}_{\infty}(\Sigma, \alpha) \geq$ $\varrho_{4}(\Sigma) \geq 3$.

Then the remaining arguments consist of two steps according to the value of $\varrho_{4}(\Sigma)$.

Step 1. $\varrho_{4}(\Sigma) \geq 4$.

In this step, from Definition 3.2, we know all the closed characteristics satisfy

$$
i\left(y_{j}, 1\right)+2 S^{+}\left(y_{j}\right)-v\left(y_{j}, 1\right) \geq 4, \quad 1 \leq j \leq 4 .
$$

By Theorem 3.1 and (3.5)-(3.9), (3.14), we obtain

$$
\begin{aligned}
i\left(y_{j}^{2 m_{j}}\right) & \geq 2 T-8, \\
i\left(y_{j}^{2 m_{j}}\right)+v\left(y_{j}^{2 m_{j}}\right)-1 & \leq 2 T-2, \\
i\left(y_{j}^{2 m_{j}+m}\right) & \geq 2 T, \quad \forall m \geq 1, \\
i\left(y_{j}^{2 m_{j}-m}\right)+v\left(y_{j}^{2 m_{j}-m}\right)-1 & \leq 2 T-9, \quad \forall m \geq 1 .
\end{aligned}
$$

Hence by (3.17)-(3.18) and Proposition 2.3, we have

$$
C_{S^{1}, 2 T-2 l}\left(\Psi_{a}, S^{1} \cdot u_{j}^{m}\right)=0, \forall m \neq 2 m_{j}, 1 \leq j \leq 4, \quad 1 \leq l \leq 4,
$$

where $u_{j}$ is the critical point of $\Psi_{a}$ corresponding to $y_{j}$. 
By (3.19) and Propositions 2.11, 2.12, we can find $p, q, r, t \in\{1,2,3,4\}$ such that

$$
\begin{aligned}
& \Phi^{\prime}\left(u_{p}^{2 m_{p}}\right)=0, \quad \Phi\left(u_{p}^{2 m_{p}}\right)=c_{T-3}, \quad C_{S^{1}, 2 T-8}\left(\Psi_{a}, S^{1} \cdot u_{p}^{2 m_{p}}\right) \neq 0, \\
& \Phi^{\prime}\left(u_{q}^{2 m_{q}}\right)=0, \quad \Phi\left(u_{q}^{2 m_{q}}\right)=c_{T-2}, \quad C_{S^{1}, 2 T-6}\left(\Psi_{a}, S^{1} \cdot u_{q}^{2 m_{q}}\right) \neq 0 \\
& \Phi^{\prime}\left(u_{r}^{2 m_{r}}\right)=0, \quad \Phi\left(u_{r}^{2 m_{r}}\right)=c_{T-1}, \quad C_{S^{1}, 2 T-4}\left(\Psi_{a}, S^{1} \cdot u_{r}^{2 m_{r}}\right) \neq 0 \\
& \Phi^{\prime}\left(u_{t}^{2 m_{t}}\right)=0, \quad \Phi\left(u_{t}^{2 m_{t}}\right)=c_{T}, \quad C_{S^{1}, 2 T-2}\left(\Psi_{a}, S^{1} \cdot u_{t}^{2 m_{t}}\right) \neq 0,
\end{aligned}
$$

where we denote also by $u_{p}^{2 m_{p}}, u_{q}^{2 m_{q}}, u_{r}^{2 m_{r}}$ and $u_{t}^{2 m_{t}}$ the corresponding critical points of $\Phi$. By Proposition 2.9, we have $c_{T-3}<c_{T-2}<c_{T-1}<c_{T}$. Hence $p, q, r$ and $t$ are pairwise distinct.

On the other hand, we have $y_{3}=-y_{4}$ as supposed before. Then by Lemma 3.4, we obtain $2 m_{3}=2 m_{4}$ and $\Phi\left(u_{3}^{2 m_{3}}\right)=\Phi\left(u_{4}^{2 m_{4}}\right)$, which is a contradiction. We complete the proof of Theorem 1.1 in this step.

Step 2. $\varrho_{4}(\Sigma)=3$.

In this step, as before, we suppose that $\left(\tau_{j}, y_{j}\right)_{1 \leq j \leq 2}$ are symmetric. So we have $i\left(y_{j}, 1\right)+2 S^{+}\left(y_{j}\right)-v\left(y_{j}, 1\right)<4$ for $j=3,4$ by Definition 3.2 and Lemma 3.6. More precisely, by p. 27 of [16], we have the following two cases:

Case A For $j=3,4, i\left(y_{j}, 1\right)=4$ and the matrix $M_{j}$ can be connected within $\Omega^{0}\left(M_{j}\right)$ to $N_{1}(1,-1)^{\diamond 2} \diamond M^{\prime}$ for some $M^{\prime} \in \operatorname{Sp}(2)$ and $M^{\prime} \in\left\{R(\theta), D(\lambda), N_{1}(-1, b)\right.$, $\left.I_{2}\right\}$, where $b \in\{ \pm 1,0\}, \lambda= \pm 2$, and $R(\theta), D(\lambda), N_{1}(-1, b)$ are basic normal forms defined in Definition 1.8.9 of [9].

Case B For $j=3,4, i\left(y_{j}, 1\right)=5$ and the matrix $M_{j}$ can be connected within $\Omega^{0}\left(M_{j}\right)$ to $N_{1}(1,-1)^{\diamond 3}$.

In both cases, we have

$$
i\left(y_{j}, 1\right)+2 S_{\gamma\left(\tau_{j}\right)}^{+}(1)-v\left(y_{j}, 1\right)=3, \quad j=3,4 .
$$

Then by Theorem 3.1, (3.5)-(3.9) and (3.20), we obtain

$$
\begin{aligned}
& i\left(y_{j}^{2 m_{j}}\right) \geq 2 T-8, \quad 1 \leq j \leq 4, \\
& i\left(y_{j}^{2 m_{j}}\right)+v\left(y_{j}^{2 m_{j}}\right)-1 \leq 2 T-2, \quad 1 \leq j \leq 4, \\
& i\left(y_{j}^{2 m_{j}+m}\right) \geq 2 T, \quad \forall m \geq 1, \quad 1 \leq j \leq 4, \\
& i\left(y_{j}^{2 m_{j}-1}\right)+v\left(y_{j}^{2 m_{j}-1}\right)-1=2 T-8, \quad 3 \leq j \leq 4, \\
& i\left(y_{j}^{2 m_{j}-m}\right)+v\left(y_{j}^{2 m_{j}-m}\right)-1<2 T-8, \quad \forall m \geq 2, \quad 3 \leq j \leq 4, \\
& i\left(y_{j}^{2 m_{j}-m}\right)+v\left(y_{j}^{2 m_{j}-m}\right)-1<2 T-8, \quad \forall m \geq 1, \quad 1 \leq j \leq 2,
\end{aligned}
$$

Thus by Propositions 2.11 and 2.12, we can find $\left(j_{k}, l_{j_{k}}\right)_{1 \leq k \leq 4}$ such that

$$
\Phi^{\prime}\left(u_{j_{k}}^{l_{j_{k}}}\right)=0, \quad \Phi\left(u_{j_{k}}^{l_{j_{k}}}\right)=c_{T+1-k}, \quad C_{S^{1}, 2 T-2 k}\left(\Psi_{a}, S^{1} \cdot u_{j_{k}}^{l_{j_{k}}}\right) \neq 0
$$


where we denote by $u_{j_{k}}^{l_{j_{k}}}$ the corresponding critical points of $\Phi$ (or $\Psi_{a}$ ). Note that by Proposition 2.9, the numbers $c_{T+1-k}$ for $1 \leq k \leq 4$ are pairwise distinct critical values of $\Phi$. Then for $1 \leq k \neq k^{\prime} \leq 4$, if $j_{k}=j_{k^{\prime}}$, the times of iteration must be different, i.e., $l_{j_{k}} \neq l_{j_{k^{\prime}}}$. Hence we have $\left(j_{k}, l_{j_{k}}\right)=\left(j_{k}, 2 m_{j_{k}}\right)$ for $1 \leq k \leq 3$ and $j_{1}, j_{2}, j_{3}$ are pairwise distinct, and so $\left\{j_{1}, j_{2}, j_{3}\right\}=\{1,2,3\}$ or $\left\{j_{1}, j_{2}, j_{3}\right\}=\{1,2,4\}$. In fact, by Proposition 2.3 and (3.23)-(3.26), we have $C_{S^{1}, 2 T-2 k}\left(\Psi_{a}, S^{1} \cdot u_{j}^{m}\right)=0$ for $1 \leq k \leq 3,1 \leq j \leq 4$ and any integer $m \neq 2 m_{j}$. Thus $l_{j_{k}}=2 m_{j_{k}}$, and then $\Phi\left(u_{j_{k}}^{2 m_{j_{k}}}\right)=c_{T+1-k}$ for $1 \leq k \leq 3$ by (3.27), $j_{1}, j_{2}, j_{3}$ are pairwise distinct. By Lemma 3.4 (the same argument as in the end of Step 1), we know the numbers 3 and 4 can not belong to $\left\{j_{1}, j_{2}, j_{3}\right\}$ simultaneously, hence $\left\{j_{1}, j_{2}, j_{3}\right\}=\{1,2,3\}$ or $\left\{j_{1}, j_{2}, j_{3}\right\}=\{1,2,4\}$.

Note that we have $l_{j_{4}}=2 m_{j_{4}}-1$ and

$$
\Phi^{\prime}\left(u_{j_{4}}^{2 m_{j_{4}}-1}\right)=0, \quad \Phi\left(u_{j_{4}}^{2 m_{j_{4}}-1}\right)=c_{T-3}, \quad C_{S^{1}, 2 T-8}\left(\Psi_{a}, S^{1} \cdot u_{j_{4}}^{2 m_{j_{4}}-1}\right) \neq 0
$$

for any $j_{4} \in\{3,4\}$. In fact, we have $l_{j_{4}} \in\left\{2 m_{j_{4}}-1,2 m_{j_{4}}\right\} \equiv \Delta$ since we have

$$
C_{S^{1}, 2 T-8}\left(\Psi_{a}, S^{1} \cdot u_{j_{4}}^{m}\right)=0
$$

for $m \notin \Delta$ by (3.23), (3.25), (3.26) and Proposition 2.3. On the other hand, since $\left\{j_{1}, j_{2}, j_{3}\right\}=\{1,2,3\}$ or $\left\{j_{1}, j_{2}, j_{3}\right\}=\{1,2,4\}$, and using Lemma 3.4, we have $\Phi\left(u_{j_{4}}^{2 m_{j_{4}}}\right)=\Phi\left(u_{j_{k}}^{2 m_{j_{k}}}\right)$ for some $1 \leq k \leq 3$. This implies $\Phi\left(u_{j_{4}}^{2 m_{j_{4}}}\right)=c_{T+1-k}$ and $\Phi\left(u_{j_{4}}^{l_{j_{4}}}\right)=c_{T+1-4}$ by (3.27). Hence $l_{j_{4}}=2 m_{j_{4}}-1$ by Proposition 2.9 , and then (3.28) holds by (3.26), (3.27) and Proposition 2.3.

Note that a commutative property for closed characteristics in the common index jump intervals was first discovered in [16] and was crucial for the proof of the existence of at least four geometrically distinct closed characteristics on every compact convex hypersurface in $\mathbf{R}^{8}$. This property is also important for our proof of Theorem 1.1. Now we recall it as follows.

Definition 3.7 For any tuple $\left(T, m_{1}, m_{2}, m_{3}\right)$ found by the common index jump theorem and $j_{1}, j_{2}, j_{3}$ satisfying (3.21)-(3.27), we define its common index jump interval to be $[2 T-6,2 T-2]$. For any even integer $2 T-2 s \in[2 T-6,2 T-2]$, noticing that $u_{3}$ and $u_{4}$ have the same index iterations, nullities, critical values, and critical module properties by Lemma 3.4, thus we let $\xi_{T}(s) \in\{1,2,3\}$ be the unique integer satisfying $c_{T+1-s}=\Phi\left(u_{\xi_{T}(s)}^{2 m_{\xi_{T}(s)}}\right)$, i.e., we have $\xi_{T}(s)=j_{s}$.

Let $v \in \mathbf{R}^{h}$ be the vector given by (3.11) and $A(v)$ be the set given by Theorem 4.2 of [10] associated to $v$. For any $a=\left(a_{1}, \ldots, a_{h}\right) \in A(v)$, as in Theorem 4.2 of [10], let $\chi(a) \equiv\left(\psi\left(a_{1}\right), \ldots, \psi\left(a_{h}\right)\right)$, we have $-a \in A(v)$ and $\chi(a) \neq \chi(-a)$. For any tuples $(T, \chi(a))$ and $\left(T^{\prime}, \chi(-a)\right)$ satisfying (3.13), let $m_{k}=\left(\left[\frac{T}{M \hat{i}\left(y_{k}\right)}\right]+\chi(a)_{k}\right) M$ and $m_{k}^{\prime}=\left(\left[\frac{T^{\prime}}{M \hat{i}\left(y_{k}\right)}\right]+\chi(-a)_{k}\right) M$ be given by (3.10). 
By Proposition 4.5 of [16] (cf. its another statement given by Lemma 6.2 of [17]), we have the following.

Proposition 3.8 Let $1 \leq \alpha, \beta \leq 3$ and $\alpha \neq \beta$ be fixed. Then there exist an $a \in A(v)$ and $T, T^{\prime}$ satisfying (3.13) such that $\Phi\left(u_{\alpha}^{2 m_{\alpha}}\right)>\Phi\left(u_{\beta}^{2 m_{\beta}}\right)$ and $\Phi\left(u_{\alpha}^{2 m_{\alpha}^{\prime}}\right)<\Phi\left(u_{\beta}^{2 m_{\beta}^{\prime}}\right)$, where $m_{k}, m_{k}^{\prime}$ are given as above. In particular, we have the following diagram

$$
\begin{array}{cccc}
c_{T+1-\xi_{T}^{-1}(\beta)} & c_{T+1-\xi_{T}^{-1}(\alpha)} & c_{T^{\prime}+1-\xi_{T^{\prime}}^{-1}(\alpha)} & c_{T^{\prime}+1-\xi_{T^{\prime}}^{-1}(\beta)} \\
u_{\beta}^{2 m_{\beta}} & u_{\alpha}^{2 m_{\alpha}} & u_{\alpha}^{2 m_{\alpha}^{\prime}} & 2 m_{\beta}^{\prime}
\end{array},
$$

where the first row in the diagram is the critical values corresponding to the critical points of $\Phi$ in the second row. Furthermore, we have $2 T-2 \xi_{T}^{-1}(\beta)<2 T-2 \xi_{T}^{-1}(\alpha)$ and $2 T^{\prime}-2 \xi_{T^{\prime}}^{-1}(\beta)>2 T^{\prime}-2 \xi_{T^{\prime}}^{-1}(\alpha)$, i.e., the orders of the two closed characteristics in the common index jump intervals interchanged.

Now we fix a tuple $\left(T^{*}, m_{1}^{*}, m_{2}^{*}, m_{3}^{*}\right)$ and $\left(j_{k}^{*}, l_{j_{k}^{*}}^{*}\right)$ for $1 \leq k \leq 4$ satisfying (3.21)-(3.27). Since $j_{4}^{*} \in\{3,4\}$, by Lemma 3.4, we may assume $j_{4}^{*}=3$ without loss of generality. In the following, we separate the proof of Theorem 1.1 into several subcases according to Cases A and B. Suppose $\left(T, m_{1}, m_{2}, m_{3}, m_{4}\right)$ be any tuple and $\left(j_{k}, l_{j_{k}}\right)$ satisfy $(3.21)-(3.27)$.

(i) If $\left(\tau_{3}, y_{3}\right)$ belongs to Case B.

By the proof of Lemma 5.1 of [16], we have $i\left(y_{3}^{2 m_{3}}\right)+v\left(y_{3}^{2 m_{3}}\right)-1=2 T-2$ and

$$
\begin{aligned}
& \operatorname{rank} C_{S^{1}, 2 T-2}\left(\Psi_{a}, S^{1} \cdot u_{3}^{2 m_{3}}\right) \\
& \quad=k_{\nu\left(u_{3}^{2 m_{3}}\right)-1}\left(u_{3}^{2 m_{3}}\right)=k_{v\left(u_{3}\right)-1}\left(u_{3}\right)=k_{v\left(u_{3}^{2 m_{1}^{*}-1}\right)-1}\left(u_{3}^{2 m_{3}^{*}-1}\right) \\
& \quad=\operatorname{rank} C_{S^{1}, 2 T^{*-8}}\left(\Psi_{a}, S^{1} \cdot u_{3}^{2 m_{3}^{*}-1}\right) \neq 0 .
\end{aligned}
$$

Then $u_{3}^{2 m_{3}}$ is a local maximum of $\Psi_{a}$ in the local characteristic manifold of $W\left(u_{3}^{2 m_{3}}\right)$ and $\Phi\left(u_{3}^{2 m_{3}}\right)>\Phi\left(u_{i}^{2 m_{i}}\right)$ for $i=1,2$, which contradicts to Proposition 3.8.

(ii) If $\left(\tau_{3}, y_{3}\right)$ belongs to Case A and the matrix $M_{3}$ can be connected within $\Omega^{0}\left(M_{3}\right)$ to $N_{1}(1,-1)^{\diamond 2} \diamond M^{\prime}$ with $M^{\prime} \in \mathrm{Sp}(2)$ and $\sigma\left(M^{\prime}\right) \cap \mathbf{U}=\emptyset$.

By the proof of Lemma 5.2 of [16], we have $i\left(y_{3}^{2 m_{3}}\right)+v\left(y_{3}^{2 m_{3}}\right)-1=2 T-3$ and

$$
\begin{aligned}
& \operatorname{rank} C_{S^{1}, 2 T-3-l}\left(\Psi_{a}, S^{1} \cdot u_{3}^{2 m_{3}}\right)=k_{\nu\left(u_{3}^{2 m_{3}}\right)-1-l}\left(u_{3}^{2 m_{3}}\right)=k_{v\left(u_{3}^{2}\right)-1-l}\left(u_{3}^{2}\right), \\
& k_{v\left(u_{3}\right)-1}\left(u_{3}\right)=k_{v\left(u_{3}^{2 m_{3}^{*}-1}\right)-1}\left(u_{3}^{2 m_{3}^{*}-1}\right)=\operatorname{rank} C_{S^{1}, 2 T^{*-8}}\left(\Psi_{a}, S^{1} \cdot u_{3}^{2 m_{3}^{*}-1}\right) \neq 0 .
\end{aligned}
$$

Then $u_{3}$ is a local maximum of $\Psi_{a}$ in the local characteristic manifold of $W\left(u_{3}\right)$. By Proposition 2.5, we have $k_{l, \pm 1}\left(u_{3}\right)=k_{l, \pm 1}\left(u_{3}^{2}\right)$. By Proposition 2.3 and Definition 2.4, we have $k_{l}\left(u_{3}^{2}\right)=k_{l,-1}\left(u_{3}^{2}\right)$ and $k_{l}\left(u_{3}\right)=k_{l,+1}\left(u_{3}\right)$ for $l \in \mathbf{Z}$. Hence, we have 
$k_{l}\left(u_{3}^{2}\right)=0$ for $l \neq 2$ and $C_{S^{1}, 2 T-3-l}\left(\Psi_{a}, S^{1} \cdot u_{3}^{2 m_{3}}\right)=0$ for $l \neq 0$, which contradicts to $(3.27)$.

(iii) If $\left(\tau_{3}, y_{3}\right)$ belongs to Case $\mathrm{A}$ and the matrix $M_{3}$ can be connected within $\Omega^{0}\left(M_{3}\right)$ to $N_{1}(1,-1)^{\diamond 2} \diamond R(\theta)$ with $\frac{\theta}{\pi} \in \mathbf{R} \backslash \mathbf{Q}$.

In this case, by Theorem 1.3 of [8], we have

$$
\begin{gathered}
i\left(y_{3}^{m}\right)=m\left(i\left(y_{3}, 1\right)+1-1\right)+2 E\left(\frac{m \theta}{2 \pi}\right)-2-4=4 m+2 E\left(\frac{m \theta}{2 \pi}\right)-6, \\
v\left(y_{3}^{m}\right)=3, \quad \forall m \in \mathbf{N} .
\end{gathered}
$$

Adopting the notation of (3.12), by the proof of Lemma 5.3 of [16], we have

$$
i\left(y_{3}^{2 m_{3}}\right)+v\left(y_{3}^{2 m_{3}}\right)-1= \begin{cases}2 T-2 & \text { if } \quad\left(\chi_{3}, \chi_{3,1}\right)=(1,0), \\ 2 T-4 & \text { if } \quad\left(\chi_{3}, \chi_{3,1}\right)=(0,1),\end{cases}
$$

and as the above cases (i) and (ii), we have

$$
\operatorname{rank} C_{S^{1}, l}\left(\Psi_{a}, S^{1} \cdot u_{3}^{2 m_{3}}\right)=0
$$

for any integer $l \neq i\left(y_{3}^{2 m_{3}}\right)+v\left(y_{3}^{2 m_{3}}\right)-1$. Hence by (3.27), we have

$$
\Phi\left(u_{3}^{2 m_{3}}\right)=\left\{\begin{array}{cl}
c_{T} & \text { if } \quad\left(\chi_{3}, \chi_{3,1}\right)=(1,0), \\
c_{T-1} & \text { if } \quad\left(\chi_{3}, \chi_{3,1}\right)=(0,1)
\end{array}\right.
$$

and then as Case A and Case B in the proof of Lemma 5.3 of [16], we obtain a contradiction.

(iv) If $\left(\tau_{3}, y_{3}\right)$ belongs to Case $\mathrm{A}$ and the matrix $M_{3}$ can be connected within $\Omega^{0}\left(M_{3}\right)$ to $N_{1}(1,-1)^{\diamond 2} \diamond R(\theta)$ with $\frac{\theta}{\pi} \in(0,2) \cap \mathbf{Q}$.

By the proof of Lemma 5.6 of [16], we have $C_{S^{1}, 2 T^{*-2-l}}\left(\Psi_{a}, S^{1} \cdot u_{3}^{2 m_{3}^{*}}\right) \neq 0$ for some $l \in\{0,2,4\}$. When $C_{S^{1}, 2 T^{*-2}}\left(\Psi_{a}, S^{1} \cdot u_{3}^{2 m_{3}^{*}}\right) \neq 0$ or $C_{S^{1}, 2 T^{*-6}-}\left(\Psi_{a}, S^{1}\right.$. $\left.u_{3}^{2 m_{3}^{*}}\right) \neq 0$, we get a contradiction to Proposition 3.8. When $C_{S^{1}, 2 T^{*-4}}\left(\Psi_{a}, S^{1}\right.$. $\left.u_{3}^{2 m_{3}^{*}}\right) \neq 0$, we have

$$
C_{S^{1}, 2 T-2}\left(\Psi_{a}, S^{1} \cdot u_{3}^{2 m_{3}}\right)=0, \quad C_{S^{1}, 2 T-6}\left(\Psi_{a}, S^{1} \cdot u_{3}^{2 m_{3}}\right)=0
$$

and by Claim 1 in Lemma 5.6 of [16], there exist two tuples $\left(T, m_{1}, m_{2}, m_{3}\right)$ and $\left(T^{\prime}, m_{1}^{\prime}, m_{2}^{\prime}, m_{3}^{\prime}\right)$ satisfying (3.21)-(3.26) such that $c_{T}=\Phi\left(u_{1}^{2 m_{1}}\right), c_{T-2}=\Phi\left(u_{2}^{2 m_{2}}\right)$ and $c_{T^{\prime}}=\Phi\left(u_{2}^{2 m_{2}^{\prime}}\right), c_{T^{\prime}-2}=\Phi\left(u_{1}^{2 m_{1}^{\prime}}\right)$, i.e., we have the following diagram

$$
\begin{array}{llllll}
c_{T-2} & c_{T-1} & c_{T} & c_{T^{\prime}-2} & c_{T^{\prime}-1} & c_{T^{\prime}} \\
u_{2}^{2 m_{2}} & u_{3}^{2 m_{3}} & u_{1}^{2 m_{1}} & u_{1}^{2 m_{1}^{\prime}} & u_{3}^{2 m_{3}^{\prime}} & u_{2}^{2 m_{2}^{\prime}},
\end{array},
$$

where the first row in the diagram is the critical values corresponding to the critical points of $\Phi$ on the second row. This implies that the orders of closed characteristics 
in the common index jump intervals have some commutative properties. Then by the proof of Claim 2 in Lemma 5.6 of [16], The matrix $M_{1}, M_{2}$ can be connected within $\Omega^{0}\left(M_{1}\right), \Omega^{0}\left(M_{2}\right)$ to $R\left(\vartheta_{1}\right) \diamond R\left(\vartheta_{2}\right) \diamond M_{1}^{\prime}$ and $R\left(\varphi_{1}\right) \diamond R\left(\varphi_{2}\right) \diamond M_{2}^{\prime}$, respectively, with $\frac{\vartheta_{i}}{\pi}, \frac{\varphi_{i}}{\pi} \in \mathbf{R} \backslash \mathbf{Q}$ for $i=1,2$ and $M_{1}^{\prime}, M_{2}^{\prime} \in \operatorname{Sp}(2)$. Moreover, $M_{1}^{\prime}, M_{2}^{\prime} \in$ $\left\{I_{2}, N_{1}(1,-1),-I_{2}, N_{1}(-1,1), R(\vartheta)\right\}$, and we have

$$
i\left(y_{1}^{2 m_{1}}\right)+v\left(y_{1}^{2 m_{1}}\right)-1=2 T-2, \quad C_{S^{1}, 2 T-2-l}\left(\Psi_{a}, S^{1} \cdot u_{1}^{2 m_{1}}\right)=0, \quad \forall l \neq 0,
$$

i.e., $u_{1}^{2 m_{1}}$ is a local maximum in the local characteristic manifold $W\left(u_{1}^{2 m_{1}}\right)$, and also

$$
i\left(y_{2}^{2 m_{2}}\right)+v\left(y_{2}^{2 m_{2}}\right)-1=2 T-6, \quad C_{S^{1}, 2 T-6-l}\left(\Psi_{a}, S^{1} \cdot u_{2}^{2 m_{2}}\right)=0, \quad \forall l \neq 0,
$$

i.e., $u_{2}^{2 m_{2}}$ is a local maximum in the local characteristic manifold $W\left(u_{2}^{2 m_{2}}\right)$. Thus we have

$$
\begin{aligned}
& \operatorname{rank} C_{S^{1}, 2 T-2}\left(\Psi_{a}, S^{1} \cdot u_{1}^{2 m_{1}}\right)=1 \\
& \operatorname{rank} C_{S^{1}, 2 T-6}\left(\Psi_{a}, S^{1} \cdot u_{2}^{2 m_{2}}\right)=1
\end{aligned}
$$

And by Claim 3 in Lemma 5.6 of [16], We have $C_{S^{1}, 2 k+1}\left(\Psi_{a}, S^{1} \cdot u_{j}^{m}\right)=0$ for $k \in \mathbf{Z}$, $m \in \mathbf{N}$ and $j=1,2$. Hence we have

$$
\begin{aligned}
M_{2 T-6} & =\sum_{1 \leq j \leq 4, p \in \mathbf{N}} \operatorname{rank} C_{S^{1}, 2 T-6}\left(\Psi_{a}, S^{1} \cdot u_{j}^{p}\right) \\
& =\operatorname{rank} C_{S^{1}, 2 T-6}\left(\Psi_{a}, S^{1} \cdot u_{2}^{2 m_{2}}\right)=1=b_{2 T-6}, \\
M_{2 T-2} & =\sum_{1 \leq j \leq 4, p \in \mathbf{N}} \operatorname{rank} C_{S^{1}, 2 T-2}\left(\Psi_{a}, S^{1} \cdot u_{j}^{p}\right) \\
& =\operatorname{rank} C_{S^{1}, 2 T-2}\left(\Psi_{a}, S^{1} \cdot u_{1}^{2 m_{1}}\right)=1=b_{2 T-2} .
\end{aligned}
$$

In fact, the second equality in (3.34) follows from (3.23)-(3.26), (3.29), (3.30), Lemma 3.4 and Proposition 2.3; the second equality in (3.35) follows from (3.23)(3.26), (3.29), (3.31), Lemma 3.4 and Proposition 2.3. The third equality in (3.34) and (3.35) follows from (3.33) and (3.32), respectively. The last equalities in (3.34) and (3.35) follow from Theorem 2.8 .

Hence by Theorem 2.8, we have

$$
\begin{aligned}
& M_{2 T-2}-M_{2 T-3}+\cdots-M_{1}+M_{0} \geq b_{2 T-2}-b_{2 T-3}+\cdots-b_{1}+b_{0}, \\
& M_{2 T-3}-M_{2 T-4}+\cdots+M_{1}-M_{0} \geq b_{2 T-3}-b_{2 T-4}+\cdots+b_{1}-b_{0}, \\
& M_{2 T-6}-M_{2 T-7}+\cdots-M_{1}+M_{0} \geq b_{2 T-6}-b_{2 T-7}+\cdots-b_{1}+b_{0}, \\
& M_{2 T-7}-M_{2 T-8}+\cdots+M_{1}-M_{0} \geq b_{2 T-7}-b_{2 T-8}+\cdots+b_{1}-b_{0} .
\end{aligned}
$$


Thus from (3.34) and (3.35), we have

$$
\begin{aligned}
& M_{2 T-3}-M_{2 T-4}+\cdots+M_{1}-M_{0}=b_{2 T-3}-b_{2 T-4}+\cdots+b_{1}-b_{0}, \\
& M_{2 T-6}-M_{2 T-7}+\cdots-M_{1}+M_{0}=b_{2 T-6}-b_{2 T-7}+\cdots-b_{1}+b_{0} .
\end{aligned}
$$

Adding (3.40) with (3.41), and by Theorem 2.8 again, we have

$$
\begin{aligned}
-1 & =b_{2 T-3}-b_{2 T-4}+b_{2 T-5}=M_{2 T-3}-M_{2 T-4}+M_{2 T-5} \\
& =\sum_{\substack{2 T-3 \leq q \leq 2 T-5 \\
1 \leq j \leq 4, m \in \mathbf{N}}}(-1)^{q+1} \operatorname{rank} C_{S^{1}, q}\left(\Psi_{a}, S^{1} \cdot u_{j}^{m}\right) \\
& =\sum_{\substack{2 T-3 \leq q \leq 2 T-5 \\
3 \leq j \leq 4}}(-1)^{q+1} \operatorname{rank} C_{S^{1}, q}\left(\Psi_{a}, S^{1} \cdot u_{j}^{2 m_{j}}\right) \\
& =2 \sum_{q \in \mathbf{Z}}(-1)^{q+1} \operatorname{rank} C_{S^{1}, q}\left(\Psi_{a}, S^{1} \cdot u_{3}^{2 m_{3}}\right) .
\end{aligned}
$$

Here in the next to the last equality, we have used (3.23)-(3.26), (3.30), (3.31) and Proposition 2.3. In the last equality, we have used (3.29) and Lemma 3.4. Now the right-hand side of equality (3.42) is an even number, while the left-hand side of equality (3.42) is odd, it is a contradiction.

(v) If $\left(\tau_{3}, y_{3}\right)$ belongs to Case A and the matrix $M_{3}$ can be connected within $\Omega^{0}\left(M_{3}\right)$ to $N_{1}(1,-1)^{\diamond 2} \diamond N_{1}(-1, b)$ with $b=0,-1$. Note that the case $N_{1}(-1,0)=-I_{2}$ has already been proved in the above case (iv) since $-I_{2}=R(\pi)$. And the proof for $R(\pi)$ also applies to the case $N_{1}(-1,-1)$, since the index iteration formulae for $-I_{2}$ and $N_{1}(-1,-1)$ are the same by Theorem 1.3 of [8], the only difference is their nullities for $2 m$-th iteration, but this will not affect our argument. In fact, we replace $v\left(y_{j}^{2 m}\right)$ by $v\left(y_{j}^{2 m}\right)+1$ with $j=3,4$, in all the corresponding formulae, and the proof goes through as in Case (iv).

(vi) If $\left(\tau_{3}, y_{3}\right)$ belongs to Case $\mathrm{A}$ and the matrix $M_{3}$ can be connected within $\Omega^{0}\left(M_{3}\right)$ to $N_{1}(1,-1)^{\diamond 2} \diamond N_{1}(-1,1)$.

By the proof of Lemma 5.8 of [16], we have $C_{S^{1}, 2 T^{*-2-l}}\left(\Psi_{a}, S^{1} \cdot u_{3}^{2 m_{3}^{*}}\right) \neq 0$ for some $l \in\{0,2\}$. When $C_{S^{1}, 2 T^{*}-2}\left(\Psi_{a}, S^{1} \cdot u_{3}^{2 m_{3}^{*}}\right) \neq 0$, we get a contradiction to Proposition 3.8. When $C_{S^{1}, 2 T^{*-4}}\left(\Psi_{a}, S^{1} \cdot u_{3}^{2 m_{3}^{*}}\right) \neq 0$, the Claims $1-4$ in Lemma 5.6 of [16] could transplant to the case (ii) of Lemma 5.8 of [16]. Then we also get a contradiction as in case (iv).

(vii) If $\left(\tau_{3}, y_{3}\right)$ belongs to Case $\mathrm{A}$ and the matrix $M_{3}$ can be connected within $\Omega^{0}\left(M_{3}\right)$ to $N_{1}(1,-1)^{\diamond 2} \diamond I_{2}$. As the above case (i), by the same proof of Lemma 5.9 of [16], we obtain a contradiction to Proposition 3.8.

Combining cases (i)-(vii), we get contradictions for all the possible cases in Step 2. The proof of Theorem 1.1 is complete.

Acknowledgments Hui Liu Partially supported by NSFC (No. 11401555), China Postdoctoral Science Foundation No. 2014T70589, CUSF (No. WK0010000037). Yiming Long Partially supported by NSFC 
(No. 11131004), MCME and LPMC of Ministry of Education of China, Nankai University, and the Beijing Center for Math. and Info. Inter. Sciences at Capital Normal University. Wei Wang Partially supported by National Natural Science Foundation of China No. 11222105, Foundation for the Author of National Excellent Doctoral Dissertation of PR China No. 201017. Ping'an Zhang Partially supported by the Fundamental Research Funds for the Central Universities (No. K5051270011).

\section{References}

1. Ekeland, I.: Une théorie de Morse pour les systèmes hamiltoniens convexes. Ann. IHP. Anal. non Linéaire. 1, 19-78 (1984)

2. Ekeland, I.: Convexity Methods in Hamiltonian Mechanics. Springer, Berlin (1990)

3. Ekeland, I., Hofer, H.: Convex Hamiltonian energy surfaces and their closed trajectories. Commun. Math. Phys. 113, 419-467 (1987)

4. Ekeland, I., Lassoued, L.: Multiplicité des trajectoires fermées d'un systéme hamiltonien sur une hypersurface d'energie convexe. Ann. IHP. Anal. non Linéaire. 4, 1-29 (1987)

5. Fadell, E., Rabinowitz, P.: Generalized cohomological index theories for Lie group actions with an application to bifurcation questions for Hamiltonian systems. Invent. Math. 45(2), 139-174 (1978)

6. Hofer, H., Wysocki, K., Zehnder, E.: The dynamics on three-dimensional strictly convex energy surfaces. Ann. Math. 148, 197-289 (1998)

7. Liu, C., Long, Y., Zhu, C.: Multiplicity of closed characteristics on symmetric convex hypersurfaces in $\mathbf{R}^{2 n}$. Math. Ann. 323, 201-215 (2002)

8. Long, Y.: Precise iteration formulae of the Maslov-type index theory and ellipticity of closed characteristics. Adv. Math. 154, 76-131 (2000)

9. Long, Y.: Index Theory for Symplectic Paths with Applications. Progress in Math. 207. Birkhäuser, Basel (2002)

10. Long, Y., Zhu, C.: Closed characteristics on compact convex hypersurfaces in $\mathbf{R}^{2 n}$. Ann. Math. 155, 317-368 (2002)

11. Rabinowitz, P.H.: Periodic solutions of Hamiltonian systems. Commun. Pure Appl. Math. 31, 157-184 (1978)

12. Szulkin, A.: Morse theory and existence of periodic solutions of convex Hamiltonian systems. Bull. Soc. Math. Fr. 116, 171-197 (1988)

13. Wang, W.: Stability of closed characteristics on compact convex hypersurfaces in $\mathbf{R}^{6}$. J. Eur. Math. Soc. 11, 575-596 (2009)

14. Wang, W.: Symmetric closed characteristics on symmetric compact convex hypersurfaces in $\mathbf{R}^{2 n}$. J. Diff. Equ. 246, 4322-4331 (2009)

15. Wang, W.: Closed trajectories on symmetric convex Hamiltonian energy surfaces. Discrete Contin. Dyn. Syst. 32(2), 679-701 (2012)

16. Wang, W.: Closed characteristics on compact convex hypersurfaces in $\mathbf{R}^{8}$, arXiv:1305.4680v2

17. Wang, W.: Irrationally elliptic closed characteristics on compact convex hypersurfaces in $\mathbf{R}^{6}$. J. Funct. Anal. 267, 799-841 (2014)

18. Wang, W., Hu, X., Long, Y.: Resonance identity, stability and multiplicity of closed characteristics on compact convex hypersurfaces. Duke Math. J. 139(3), 411-462 (2007)

19. Weinstein, A.: Periodic orbits for convex Hamiltonian systems. Ann. Math. 108, 507-518 (1978) 